\title{
Adjuvant oxaliplatin- or irinotecan-containing chemotherapy improves overall survival following resection of metachronous colorectal liver metastases
}

\author{
Jin-Hwang Liu • Yao-Yu Hsieh • Wei-Shone Chen • Yen-Ning Hsu • Gar-Yang Chau • \\ Hao-Wei Teng • Kuang-Liang King • Tzu-Chen Lin • Chen-Hwai Tzeng • Jen-Kou Lin
}

Accepted: 10 June 2010 / Published online: 24 June 2010

(C) The Author(s) 2010. This article is published with open access at Springerlink.com

\begin{abstract}
Purpose Adjuvant systemic 5-fluorouracil (5-FU)-based chemotherapy improves survival after resection of synchronous colorectal liver metastases (CLMs), but not metachronous. We retrospectively examined if adjuvant chemotherapy with new regimen containing oxaliplatin or irinotecan improved survivals after resection of metachronous CLMs.

Methods Between 2000 and 2007, 52 patients having undertaken resection of metachronous CLMs with curative intent were identified from Taipei Veterans General Hospital hospitalization registry. One patient with perioperative mortality and another being lost to follow-up within 3 months after metastasectomy were excluded. Thirty-one patients experienced six to 12 cycles of FOLFOX or FOLFIRI chemotherapy while 19 patients with 5-FU/leucovorin (LV)-based chemotherapy following CLM
\end{abstract}

Jin-Hwang Liu, Yao-Yu Hsieh, and Wei-Shone Chen contributed equally to this work

J.-H. Liu $(\bowtie) \cdot$ Y.-Y. Hsieh $\cdot$ Y.-N. Hsu $\cdot$ H.-W. Teng $•$

C.-H. Tzeng

Division of Medical Oncology, Taipei Veterans General Hospital,

National Yang-Ming University,

No. 201, Shi-Pai Road, Section 2,

Taipei, Taiwan 112, Republic of China

e-mail: jhliu@vghtpe.gov.tw

W.-S. Chen $\cdot$ T.-C. Lin $\cdot$ J.-K. Lin

Division of Colorectal Surgery, Taipei Veterans General Hospital, National Yang-Ming University,

Taipei, Taiwan, Republic of China

W.-S. Chen

Division of Experimental Surgery,

Taipei Veterans General Hospital, National Yang-Ming University,

Taipei, Taiwan, Republic of China

G.-Y. Chau $\cdot$ K.-L. King

Division of General Surgery, Taipei Veterans General Hospital,

National Yang-Ming University,

Taipei, Taiwan, Republic of China resection. The primary end point was disease-free survival (DFS) and secondary end point, overall survival (OS).

Results By the univariate analysis, median DFS was 34.3 months in the FOLFOX/FOLFIRI group vs 14.2 months in the $5-\mathrm{FU} / \mathrm{LV}$ group $(P=0.022)$. The median OS and 5-year survival rates were longer than 57.7 months (not reached, with median follow-up of 35.5 months) and $54.0 \%$, respectively, in the FOLFOX/FOLFIRI group compared to 49 months and $34.6 \%$ in the $5-\mathrm{FU} / \mathrm{LV}$ group $(P=0.027)$. FOLFOX/FOLFIRI chemotherapy was shown by multivariate analyses to be an independent factor predicting a better DFS (hazard ratio $[\mathrm{HR}]=0.37 ; 95 \% \mathrm{CI}$ : $0.15-0.94 ; P=0.036)$ and a better OS $(\mathrm{HR}=0.27 ; 95 \% \mathrm{CI}$ : 0.083-0.86, $P=0.026$ ) than 5-FU/LV-based.

Conclusions Adjuvant FOLFOX/FOLFIRI chemotherapy following resection of metachronous CLMs is demonstrated to have better DFS and OS than 5-FU/LV chemotherapy.

Keywords Adjuvant chemotherapy . Colorectal liver metastasis · Irinotecan · Metachronous · Oxaliplatin

\section{Introduction}

The liver is the major metastatic site of colorectal cancers (CRCs) and is often the only organ affected [1]. Surgical resection has been an effective way to treat colorectal liver metastases (CLMs) and may lead to a hopeful long-term survival [2-6]. Survival benefit of CLM resection has been established with the reported 5-year survival rates ranging from $30 \%$ to $65 \%$ [2-6]. Among the many factors affecting the outcome of metastasectomy of CLMs, adjuvant chemotherapy appears to have potential impact on survivals of patients with CLMs after resection [7].

While systemic chemotherapy is frequently administered after resection of metastases, the optimal regimen remains to 
be determined [8-11]. Adjuvant chemotherapy with 5fluorouracil (5-FU) and leucovorin (LV) after resection of CLMs has been demonstrated to significantly benefit disease-free survival (DFS) but not overall survival (OS) $[7,9,11]$. Since more advanced oxaliplatin- and irinotecancontaining chemotherapies have established their roles in improving survival of patients with metastatic CRCs and also patients with stage II and III CRCs [12, 13], they may have a role in improving DFS or even OS in the adjuvant setting following metastasectomy of CLMs. Nonetheless, postoperative or perioperative adjuvant chemotherapy using oxaliplatin-containing regimens was demonstrated to have benefit in DFS but not in OS, or left OS not surveyed [7, 10, 14]. Since 5-FU-based chemotherapy following liver metastasectomy improved OS only in the synchronous group but not metachronous [15] and since most of the aforementioned data were based on heterogeneous population either with synchronous or metachronous CLMs, a possibility is thus raised that significant survival benefit of patients with metachronous CLMs after metastasectomy may need advanced adjuvant chemotherapy like oxaliplatin- or irinotecan-containing chemotherapy.

With the current surgery and adjuvant chemotherapy, 5-year survival rates of stage II and III CRCs range from $67 \%$ to $73 \%$ [12]. Most of their survival failure comes from metastases. Patients with an initial stage II or III CRC followed by a metachronous occurrence of CLMs are deemed to be able to benefit from CLM resection if the CLMs are resectable; however, the survival benefit from oxaliplatin- or irinotecancontaining adjuvant chemotherapy after resection of CLMs has not been established. We analyzed herein the impact of oxaliplatin- or irinotecan-containing adjuvant chemotherapy compared to that of 5-FU/LV-based chemotherapy on survival following resection of metachronous CLMs.

\section{Patients and methods}

\section{Identification of eligible patients}

Fifty-two consecutive patients were identified from hospitalization registry of Taipei Veterans General Hospital, Taiwan between January 1, 2000 and June, 2007 with a primary diagnosis of colorectal cancer, had their initial metachronous CLMs (diagnosis of CLMs $>6$ months after resection of primary CRCs) resected, and were treated with postoperative adjuvant chemotherapy. The CLMs were treated with a curative intent. Patients with extrahepatic metastases or macroscopic residual disease (R2 resection) were excluded. However, patients with microscopic residual disease at resection margin (R1) were allowed for recruitment. The size of the largest metastatic lesions was measured as the largest dimension on $\mathrm{CT}$ scan or MR imaging performed within
1 month prior to CLM treatment. The presence of microscopic residual tumor was determined by pathology with cut margin and lymphatic and vascular vessels in tumor-surrounding tissue being examined.

Adjuvant chemotherapy

All patients identified had ever been treated with adjuvant chemotherapy following liver metastasectomy surgery. They were given 5-FU/LV-based regimens (FU5LV2 or modified Mayo Clinc regimen [monthly 5-day bolus intravenous (IV) 5FU/LV]: $5-\mathrm{FU}$ at $400 \mathrm{mg} / \mathrm{m}^{2} /$ day and $\mathrm{LV}$ at $20 \mathrm{mg} / \mathrm{m}^{2} /$ day, IV injection from days 1 to 5 every 4 weeks for six cycles) [16, 17] or with FOLFOX or FOLFIRI $[13,17,18]$ for six to 12 cycles. The assignment of patients into the two treatment groups was at doctors' discretion while to a significant extent depending on the reimbursement of the government's insurance and probably with patients' age being taken into consideration. The majority of patients (20/26) identified after 2004 were treated with FOLFOX or FOLFIRI. Relatively more patients at age $>70$ were in the 5 -FU/LV group than in the FOLFOX/FOLFIRI group (Table 1). When FOLFOX was chosen, either FOLFOX4 or modified FOLFOX6 (oxaliplatin at $85 \mathrm{mg} / \mathrm{m}^{2}$ IV over $2 \mathrm{~h}$, day1 plus LV at $400 \mathrm{mg} / \mathrm{m}^{2}$ IV over $2 \mathrm{~h}$, plus 5 -FU at $400 \mathrm{mg} / \mathrm{m}^{2}$ IV bolus on day 1 , then $2400 \mathrm{mg} / \mathrm{m}^{2}$ continuous infusion over $46-48 \mathrm{~h}$ ) was administrated $[16,19,20]$. FOLFIRI was administrated as below: Irinotecan at $150-180 \mathrm{mg} / \mathrm{m}^{2}$ IV over $90 \mathrm{~min}$, day 1 plus $\mathrm{LV}$ at $400 \mathrm{mg} / \mathrm{m}^{2} \mathrm{IV}$ infusion to match the duration of irinotecan infusion plus 5 -FU at $400 \mathrm{mg} / \mathrm{m}^{2}$ IV bolus on day 1, then 5 -FU at $1200 \mathrm{mg} / \mathrm{m}^{2} /$ day 2-day (total of $2400 \mathrm{mg} / \mathrm{m}^{2}$ over $46-48$ h) continuous infusion [21]. Four patients had been exposed previously to oxaliplatin- or irinotecancontaining chemotherapy as adjuvant treatment after resection of primary CRC tumor, but at least 6 months apart from the studied adjuvant chemotherapy. CLMs were pathologically proven after resection of CLMs.

Statistical evaluations

DFS was calculated from the date of resection of CLMs to the date of proven recurrence, or death. For patients lost to follow-up, data were censored on the date when the patient was last seen alive without recurrence. OS was calculated from the date of CLM resection until the date of death from any cause. For patients lost to follow-up, data were censored on the date when the patient was last seen alive. DFS, OS, and 5-year survival rate were estimated by the Kaplan-Meier method. The effect difference between factors was determined by log-rank test. The factors analyzed included age, gender, origin and stage of primary tumor, maximum size of CLMs, number and distribution of CLMs, time from resection of primary CRC to diagnosis of 
CLMs, serum CEA level at CLM resection, presence or absence of microscopic residual disease at CLM resection margin, and prior adjuvant FOLFOX or FOLFIRI chemotherapy following resection of primary tumor. Factors (age, stage of primary CRC, time from surgery of primary CRC to metastasis, CLM resection margin, and serum CEA at metastasectomy) having impact on DFS with a $P$ value less than 0.1 in univariate analysis were recruited into the Cox regression model. In addition, maximum size of CLMs, number of CLMs, and time from surgery for primary CRC to metastases were also recruited as variables in the Cox proportional hazards regression models for they impacted survival significantly as reported in previous investigations
[3, 22, 23]. Hazard ratios (HRs) were presented with their 95\% confidence intervals (CIs). All statistical tests were two-sided, and $P<0.05$ was considered statistically significant. All statistical analyses were performed using SPSS 17.0 (SPSS, Chicago, IL).

\section{Results}

Patients' characteristics

Among the 52 patients identified for this study by retrospective review of their medical records, one patient
Table 1 Demographic and clinical characteristics of patients with regard to adjuvant chemotherapy following liver metastasectomy
${ }^{\text {a }} P$ values were calculated with the use of Pearson's chi-square test

\begin{tabular}{|c|c|c|c|c|}
\hline \multirow[t]{2}{*}{ Characteristic } & \multicolumn{2}{|c|}{ Adjuvant chemotherapy } & \multirow[t]{2}{*}{ Total no. } & \multirow[t]{2}{*}{$P^{\mathrm{a}}$} \\
\hline & FU/LV no. $(\%)$ & FOLFOX/FOLFIRI no. (\%) & & \\
\hline Total & $19(38)$ & $31(62)$ & $50(100)$ & \\
\hline \multicolumn{5}{|l|}{ Age (years) } \\
\hline$\leq 70$ & $6(12)$ & $20(40)$ & $26(52)$ & \multirow[t]{2}{*}{0.04} \\
\hline$>70$ & $13(26)$ & $11(22)$ & $24(48)$ & \\
\hline \multicolumn{5}{|l|}{ Gender } \\
\hline Male & $13(26)$ & $21(42)$ & $34(68)$ & \multirow[t]{2}{*}{1.0} \\
\hline Female & $6(12)$ & $10(20)$ & $16(32)$ & \\
\hline \multicolumn{5}{|l|}{ Primary tumor } \\
\hline Colon & $10(20)$ & $24(48)$ & $33(66)$ & \multirow[t]{2}{*}{0.12} \\
\hline Rectum & $9(18)$ & $7(14)$ & $16(32)$ & \\
\hline \multicolumn{5}{|c|}{ Stage of primary CRC } \\
\hline II & $8(16)$ & $11(22)$ & $19(38)$ & \multirow[t]{2}{*}{0.77} \\
\hline III & $11(22)$ & $20(40)$ & $31(62)$ & \\
\hline \multicolumn{5}{|c|}{ Maximum CLM size } \\
\hline$\leq 3 \mathrm{~cm}$ & $9(18)$ & $21(42)$ & $30(60)$ & \multirow[t]{2}{*}{0.24} \\
\hline$>3 \mathrm{~cm}$ & $10(20)$ & $10(20)$ & $20(40)$ & \\
\hline \multicolumn{5}{|c|}{ Number of CLMs } \\
\hline 1 & $10(20)$ & $19(38)$ & $29(58)$ & \multirow[t]{2}{*}{0.57} \\
\hline$\geq 2$ & $9(18)$ & $12(24)$ & $21(42)$ & \\
\hline \multicolumn{5}{|c|}{ CLM distribution } \\
\hline Unilobar & $13(26)$ & $27(54)$ & $40(80)$ & \multirow[t]{2}{*}{0.15} \\
\hline Bilobar & $6(12)$ & $4(8)$ & $10(20)$ & \\
\hline \multicolumn{5}{|c|}{ Time from surgery of primary CRC to metastasis (months) } \\
\hline$\leq 12$ & $12(24)$ & $12(24)$ & $24(48)$ & \multirow[t]{2}{*}{0.15} \\
\hline$>12$ & $7(14)$ & $19(36.7)$ & $26(52)$ & \\
\hline \multicolumn{5}{|c|}{ CEA at metastasectomy (ng/ml) } \\
\hline$\leq 50$ & $14(28)$ & $25(50)$ & $39(78)$ & \multirow[t]{2}{*}{0.73} \\
\hline$>50$ & $5(10)$ & $6(12)$ & $11(22)$ & \\
\hline \multicolumn{5}{|c|}{ CLM resection margin } \\
\hline $\mathrm{R} 0$ resection & $18(36)$ & $28(56)$ & $46(92)$ & \multirow[t]{2}{*}{0.66} \\
\hline $\mathrm{R} 1$ resection & $1(2.0)$ & $3(6.0)$ & $4(8.0)$ & \\
\hline \multicolumn{5}{|c|}{ Prior FOLFOX/FOLFIRI following resection of primary CRC } \\
\hline Yes & $0(0)$ & $4(8)$ & $4(8)$ & \multirow[t]{2}{*}{0.15} \\
\hline No & $19(38)$ & $27(54)$ & $46(92)$ & \\
\hline
\end{tabular}


was excluded from the analysis for perioperative mortality and another for being lost to follow-up within 3 months after metastasectomy. Thirty-one patients experienced six to 12 cycles of adjuvant FOLFOX or FOLFIRI chemotherapy while 19 patients with six cycles of 5-FU/LV-based chemotherapy following CLM resection.

The patients' characteristics are shown in Table 1. The median age is 70 years (range, 47 to 85 years). At the time of analysis, six patients had died, seven patients were alive with disease recurrence, and 17 patients were alive and disease-free in the FOLFOX/FOLFIRI group. The corresponding numbers in the 5-FU/LV group were eight, six, and five patients, respectively. The median size of liver metastases is $3 \mathrm{~cm}(1.2$ to $8.1 \mathrm{~cm})$. The number of CLMs was one in 29 patients, two in 19 patients, and three or more in two patients. High percentage of patients older than 70 years is associated significantly with the $5-\mathrm{FU} / \mathrm{LV}$ group (Table 1).

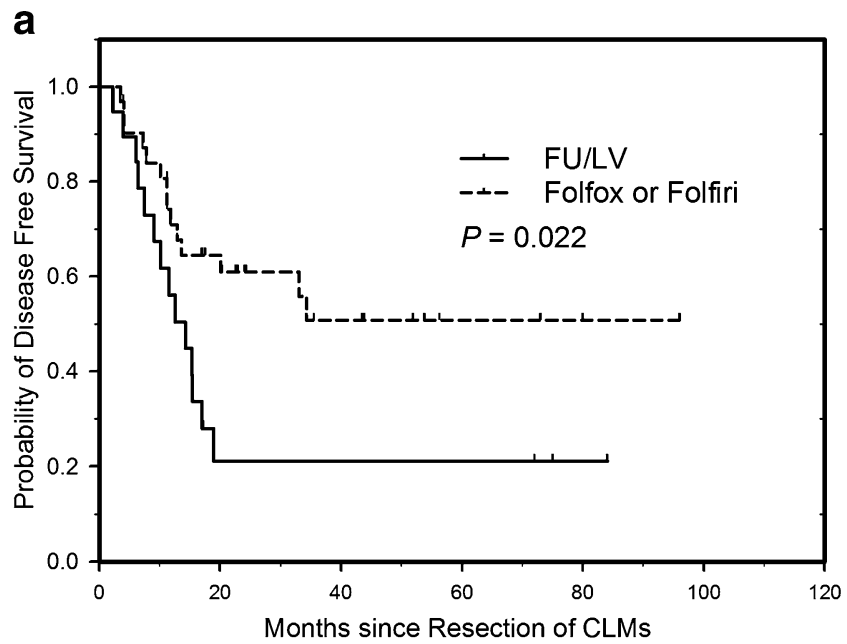

b

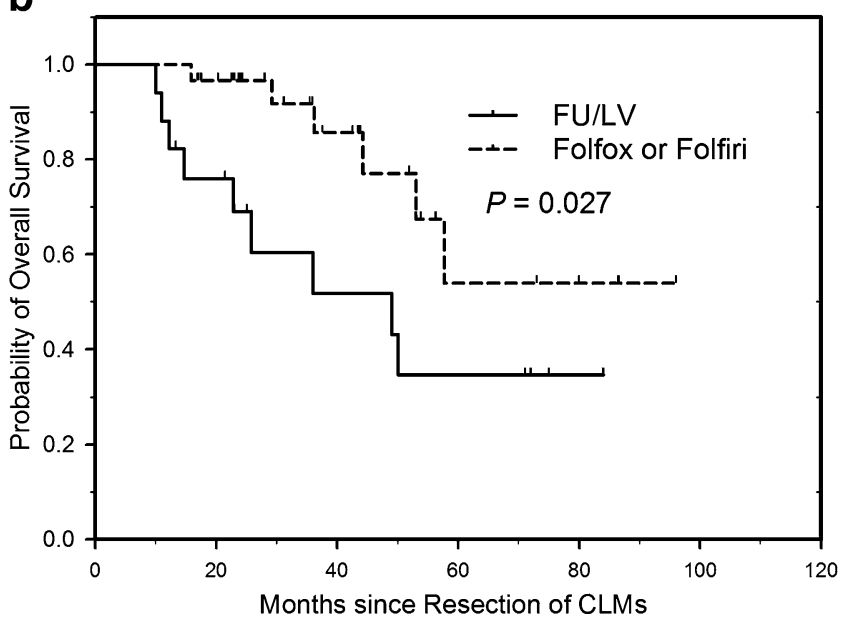

Fig. 1 Disease-free survival (a) and overall survival (b) according to adjuvant chemotherapy following resection of colorectal liver metastases. $P$ by log-rank test
Adjuvant chemotherapy

Among the 31 patients in the FOLFOX/FOLFIRI group, six patients received six cycles of FOLFOX, 11 received eight to 12 cycles of FOLFOX, five patients received six cycles of FOLFIRI, and nine patients received eight to 12 cycles of FOLFIRI. In the 5-FU/LV-based group, ten patients received six cycles of monthly 5-day bolus IV 5-FU/LV while nine patients received 12-cycle chemotherapy with FU5LV2 regimen. No chemotherapy death was recorded in this study.

Table 2 Univariate analysis associated with DFS

\begin{tabular}{|c|c|c|c|}
\hline Factors & $\begin{array}{l}\text { Patient } \\
\text { number }\end{array}$ & $\begin{array}{l}\text { Median DFS } \\
\text { (months) }\end{array}$ & $\begin{array}{l}P \\
\text { (log-rank test) }\end{array}$ \\
\hline \multicolumn{4}{|l|}{ Adjuvant chemotherapy } \\
\hline $\begin{array}{l}\text { FU/LV } \\
\text { FOLFOX/FOLFIRI }\end{array}$ & $\begin{array}{l}19 \\
31\end{array}$ & $\begin{array}{l}14.2 \\
34.3\end{array}$ & 0.022 \\
\hline \multicolumn{4}{|l|}{ Age (years) } \\
\hline $\begin{array}{l}<70 \\
\geq 70\end{array}$ & $\begin{array}{l}26 \\
24\end{array}$ & $\begin{array}{l}20.1 \\
15.3\end{array}$ & 0.391 \\
\hline \multicolumn{4}{|l|}{ Gender } \\
\hline $\begin{array}{l}\text { Male } \\
\text { Female }\end{array}$ & $\begin{array}{l}34 \\
16\end{array}$ & $\begin{array}{l}18.9 \\
17.0\end{array}$ & 0.647 \\
\hline \multicolumn{4}{|l|}{ Primary tumor } \\
\hline $\begin{array}{l}\text { Colon } \\
\text { Rectum }\end{array}$ & $\begin{array}{l}34 \\
16\end{array}$ & $\begin{array}{l}18.9 \\
15.4\end{array}$ & 0.768 \\
\hline \multicolumn{4}{|l|}{ Stage of primary CRC } \\
\hline $\begin{array}{l}\text { II } \\
\text { III }\end{array}$ & $\begin{array}{l}19 \\
31\end{array}$ & $\begin{array}{l}\text { Not achieved }(>33.0) \\
13.6\end{array}$ & 0.016 \\
\hline \multicolumn{4}{|c|}{ Maximum CLM size (cm) } \\
\hline $\begin{array}{l}\leq 3 \\
>3\end{array}$ & $\begin{array}{l}30 \\
20\end{array}$ & $\begin{array}{l}13.6 \\
33.0\end{array}$ & 0.693 \\
\hline \multicolumn{4}{|l|}{ Number of CLMs } \\
\hline $\begin{array}{l}1 \\
\geq 2\end{array}$ & $\begin{array}{l}29 \\
21\end{array}$ & $\begin{array}{l}33.0 \\
17.0\end{array}$ & 0.221 \\
\hline \multicolumn{4}{|l|}{ CLM distribution } \\
\hline $\begin{array}{l}\text { Unilobar } \\
\text { Bilobar }\end{array}$ & $\begin{array}{l}40 \\
10\end{array}$ & $\begin{array}{l}33.0 \\
15.3\end{array}$ & 0.335 \\
\hline \multicolumn{4}{|c|}{ Time from surgery of primary CRC to metastases (months) } \\
\hline $\begin{array}{l}\leq 12 \\
>12\end{array}$ & $\begin{array}{l}24 \\
26\end{array}$ & $\begin{array}{l}11.8 \\
34.3\end{array}$ & 0.036 \\
\hline \multicolumn{4}{|l|}{ CLM resection margin } \\
\hline $\begin{array}{l}\text { R0 resection } \\
\text { R1 resection }\end{array}$ & $\begin{array}{l}46 \\
4\end{array}$ & $\begin{array}{l}33.0 \\
3.9\end{array}$ & 0.00 \\
\hline \multicolumn{4}{|c|}{ CEA at metastasectomy (ng/ml) } \\
\hline $\begin{array}{l}\leq 50 \\
>50\end{array}$ & $\begin{array}{l}39 \\
11\end{array}$ & $\begin{array}{l}33.0 \\
9.0\end{array}$ & 0.003 \\
\hline \multicolumn{4}{|c|}{ Prior FOLFOX/FOLFIRI following resection of primary CRC } \\
\hline $\begin{array}{l}\text { No } \\
\text { Yes }\end{array}$ & $\begin{array}{l}46 \\
4\end{array}$ & $\begin{array}{l}17.0 \\
20.1\end{array}$ & 0.730 \\
\hline
\end{tabular}


Survival

The median follow-up time is 35.5 months (10.0 to 96 months). The median DFS was 34.3 months in the FOLFOX/FOLFIRI group compared to 14.2 months in the 5-FU/LV group (Fig. 1a). Patients in the FOLFOX/ FOLFIRI group had a favorable risk of recurrence compared to patients in the 5-FU/LV group both in univariate (Table 2) and multivariate analyses $(\mathrm{HR}=0.37$, 95\% CI: $0.15-0.94, P=0.036$ ) (Table 3). The 3 -year DFS rates were $50.8 \%$ and $21.1 \%$ in the FOLFOX/FOLFIRI and in the 5-FU/LV groups, respectively. The median OS in the 5-FU/LV group was 49 months and was not reached (>57.7 months) as of the date of analysis in the FOLFOX/ FOLFIRI group. The 3-year and 5-year OS rates were $85.7 \%$ and $54.0 \%$, respectively, in the FOLFOX/FOLFIRI group vs $51.8 \%$ and $34.6 \%$ in the $5-\mathrm{FU} / \mathrm{LV}$ group (Fig. $1 \mathrm{~b}$ ). By univariate analysis, adjuvant FOLFOX/FOLFIRI chemotherapy (vs 5-FU/LV chemotherapy), stage II primary CRC (vs stage III), negative CLM resection margin (R0 resection) (vs R1 resection) and $\leq 50 \mathrm{ng} / \mathrm{ml}$ of serum CEA at metastasectomy predicted significantly favorable DFS
(Table 2). Adjuvant FOLFOX/FOLFIRI chemotherapy and R0 resection of CLMs remained independently significant to predict favorable DFS in the multivariate analysis, and only adjuvant FOLFOX/FOLFIRI chemotherapy remained the independent significant factor to predict favorable OS in the multivariate analysis model (Table 3).

\section{Discussion}

Survival benefit from systemic adjuvant chemotherapy using either 5-FU/LV-based or oxaliplatin- or irinotecancontaining regimens following resection of CLMs has never proven definitely [7, 9-11, 14]. Subgroup analyses showed 5-FU-based chemotherapy as well as hepatic arterial infusion (HAI) of floxuridine (FUDR) chemotherapy following hepatectomy improved survival in the synchronous group, but not metachronous [15]. Contrarily, we have herein demonstrated both DFS and OS benefits from systemic FOLFOX or FOLFIRI adjuvant chemotherapy following resection of metachronous CLMs compared with 5-FU/LV-based chemotherapy. Viewing that adjuvant

Table 3 Cox regression model of the adjuvant chemotherapy with regard to disease-free and overall survivals after liver metastasectomy

\begin{tabular}{|c|c|c|c|c|c|c|}
\hline \multirow[b]{2}{*}{ Factor } & \multicolumn{3}{|c|}{ Multivariate analysis with regard to disease-free survival } & \multicolumn{3}{|c|}{ Multivariate analysis with regard to overall survival } \\
\hline & HR & $95 \% \mathrm{CI}$ & $P$ & HR & $95 \% \mathrm{CI}$ & $P$ \\
\hline \multicolumn{7}{|l|}{ Adjuvant chemotherapy } \\
\hline FU/LV & 1 & & & 1 & & \\
\hline FOLFOX/FOLFIRI & 0.37 & $0.15-0.94$ & 0.036 & 0.27 & $0.083-0.86$ & 0.026 \\
\hline \multicolumn{7}{|l|}{ Age (years) } \\
\hline$\leq 70$ & 1 & & & 1 & & \\
\hline$>70$ & 1.3 & $0.46-3.6$ & 0.64 & 2.4 & $0.60-9.3$ & 0.22 \\
\hline \multicolumn{7}{|l|}{ Stage of primary CRC } \\
\hline II & 1 & & & 1 & & \\
\hline III & 2.5 & $0.90-7.0$ & 0.081 & 3.0 & $0.76-12$ & 0.12 \\
\hline \multicolumn{7}{|c|}{ Maximum CLM size $(\mathrm{cm})$} \\
\hline$>3$ & 1 & & & 1 & & \\
\hline$\leq 3$ & 0.68 & $0.25-1.9$ & 0.45 & 0.75 & $0.19-3.0$ & 0.69 \\
\hline \multicolumn{7}{|l|}{ No. CLMs } \\
\hline 1 & 1 & & & 1 & & \\
\hline$\geq 2$ & 1.2 & $0.47-2.8$ & 0.76 & 0.81 & $0.24-2.7$ & 0.73 \\
\hline \multicolumn{7}{|c|}{ Time from surgery of primary CRC to metastases (months) } \\
\hline$\leq 12$ & 1 & & & 1 & & \\
\hline$>12$ & 0.66 & $0.29-1.5$ & 0.34 & 1.0 & $0.34-3.0$ & 0.99 \\
\hline \multicolumn{7}{|l|}{ CLM resection margin } \\
\hline R0 resection & 1 & & & 1 & & \\
\hline $\mathrm{R} 1$ resection & 5.6 & $1.2-20$ & 0.019 & 1.0 & $0.11-9.7$ & 0.99 \\
\hline \multicolumn{7}{|c|}{ CEA at metastasectomy (ng/ml) } \\
\hline$\leq 50$ & 1 & & & 1 & & \\
\hline$>50$ & 1.8 & $0.70-4.8$ & 0.21 & 2.1 & $0.59-7.3$ & 0.25 \\
\hline
\end{tabular}


postoperative or perioperative oxaliplatin-containing chemotherapy following liver metastasectomy has been shown to result in better DFS, but not OS, than 5-FU/LV-based chemotherapy [14], the most likely reason to explain the discrepancy is that patients in our study were confined to those with homogeneously metachronous CLMs instead of those with both synchronous and metachronous CLMs as in the other aforementioned studies. Whether patients with synchronous CLMs can have additive survival benefit from oxaliplatin- or irinotecan-containing adjuvant chemotherapy is unknown, although they are shown to have survival benefit from 5-FU/LV-based adjuvant chemotherapy [15]. Patients with metachronous CLMs are deemed to have better prognosis than those with synchronous CLMs $[3,22$, 24]; however, they were known to fail to have survival benefit from 5-FU/LV-based adjuvant chemotherapy [15]. Seemingly, the better the prognosis the less the benefit can be drawn from an adjuvant chemotherapy for CRCs [25]. Patient with metachronous CLMs may need oxaliplatin- or irinotecan-containing adjuvant chemotherapy following metastasectomy for a survival gain as shown in our study.

The significance of the association of patients' charactereristics with either adjuvant chemotherapy group was analyzed. As shown in Table 1, patients at age $>70$ years were significantly higher in the 5-FU/LV group. However, this may not affect the result that DFS of the 5-FU/LV group was poorer than DFS of the FOLFOX/FOLFIRI group since there is no DFS difference $(P=0.39)$ between groups of age $\leq 70$ and $>70$ in univariate analysis (Table 2), and also, age is not an independent predictor for DFS or OS (Table 3).

$\mathrm{R} 0$ resection margin (vs R1 resection) was one independent significant predictor for favorable DFS in addition to FOLFOX/FOLFIRI adjuvant chemotherapy (vs 5-FU/LVbased chemotherapy); however, it loses its independent value to predict OS in the multivariate model. One reason would be that the effect of the presence of microscopic residual tumor on DFS was offset by the long-term effect of FOLFOX/FOLFIRI chemotherapy in both the FOLFOX/ FOLFIRI and the 5-FU/LV groups once metastases recurred after adjuvant chemotherapy.

We have demonstrated the improvement of DFS, and for the first time, of OS by oxaliplatin- or irinotecan-containing adjuvant chemotherapy following resection of metachronous CLMs based on a single institute's population. To further confirm the results, prospective randomized trial is worthwhile. Given the high liver recurrence rate after metastasectomy, combination of regional HAI FUDR with systemic intravenous 5-FU has been shown to improve progressionfree survival and 2-year survival for patients after metastasectomy, not specified for synchronous or metachronous CLMs [26]. Deriving from our results, there is a potential to have survival benefit if adjuvant oxaliplatin- or irinotecancontaining therapy in combination with HAI FUDR is given following metastasectomy of either synchronous or metachronous CLMs. Actually, the potential has been demonstrated in an NCCTG/NSABP trial [27].

Acknowledgements This study was supported by grant (DOH99TD-C-111-007) from the Center of Excellence for Cancer Research at Taipei Veterans General Hospital, Taiwan and grant from the Taiwan Clinical Oncology Research Foundation.

Open Access This article is distributed under the terms of the Creative Commons Attribution Noncommercial License which permits any noncommercial use, distribution, and reproduction in any medium, provided the original author(s) and source are credited.

\section{References}

1. Stangl R, Altendorf-Hofmann A, Charnley RM et al (1994) Factors influencing the natural history of colorectal liver metastases. Lancet 343:1405-1410

2. Scheele J, Stangl R, Altendorf-Hofmann A et al (1995) Resection of colorectal liver metastases. World J Surg 19:59-71

3. Fong Y, Fortner J, Sun RL et al (1999) Clinical score for predicting recurrence after hepatic resection for metastatic colorectal cancer: analysis of 1001 consecutive cases. Ann Surg 230:309-318

4. Sugawara Y, Yamamoto J, Yamasaki S et al (2001) Estimating the prognosis of hepatic resection in patients with metastatic liver tumors from colorectal cancer with special concern for the timing of hepatectomy. Surgery 129:408-413

5. Choti MA, Sitzmann JV, Tiburi MF et al (2002) Trends in longterm survival following liver resection for hepatic colorectal metastases. Ann Surg 235:759-766

6. Adam R, Pascal G, Azoulay D et al (2003) Liver resection for colorectal metastases: the third hepatectomy. Ann Surg 238:871-883

7. Hebbar M, Pruvot FR, Romano O et al (2009) Integration of neoadjuvant and adjuvant chemotherapy in patients with resectable liver metastases from colorectal cancer. Cancer Treat Rev 35:668-675

8. Kemeny MM, Adak S, Gray B et al (2002) Combined-modality treatment for resectable metastatic colorectal carcinoma to the liver: surgical resection of hepatic metastases in combination with continuous infusion of chemotherapy - an intergroup study. J Clin Oncol 20:1499-1505

9. Portier G, Elias D, Bouche O et al (2006) Multicenter randomized trial of adjuvant fluorouracil and folinic acid compared with surgery alone after resection of colorectal liver metastases: FFCD ACHBTH AURC 9002 trial. J Clin Oncol 24:4976-4982

10. Nordlinger B, Sorbye H, Glimelius B et al (2008) Perioperative chemotherapy with FOLFOX4 and surgery versus surgery alone for resectable liver metastases from colorectal cancer (EORTC intergroup trial 40983): a randomised controlled trial. Lancet 371:1007-1016

11. Mitry E, Fields ALA, Bleiberg $\mathrm{H}$ et al (2008) Adjuvant chemotherapy after potentially curative resection of metastases from colorectal cancer: a pooled analysis of two randomized trials. J Clin Oncol 26:4906-4911

12. André T, Boni C, Navarro $M$ et al (2009) Improved overall survival with oxaliplatin, fluorouracil, and leucovorin as adjuvant treatment in stage II or III colon cancer in the MOSAIC trial. J Clin Oncol 27:3109-3116

13. Tournigand C, André T, Achille E et al (2004) FOLFIRI followed by FOLFOX6 or the reverse sequence in advanced colorectal cancer: a randomized GERCOR study. J Clin Oncol 22:229-237 
14. Kim SY, Kim HJ, Hong YS et al (2009) Resected colorectal liver metastases: does the survival differ according to postoperative chemotherapy regimen? J Surg Oncol 100:713-718

15. Wang X, Hershman DL, Abrams JA et al (2007) Predictors of survival after hepatic resection among patients with colorectal liver metastasis. Br J Cancer 97:1606-1612

16. de Gramont A, Figer A, Seymour M et al (2000) Leucovorin and fluorouracil with or without oxaliplatin as first-line treatment in advanced colorectal cancer. J Clin Oncol 18:2938-2947

17. Kelly H, Goldberg RM (2005) Systemic therapy for metastatic colorectal cancer: current options, current evidence. J Clin Oncol 23:4553-4560

18. de Gramont A, Bosset JF, Milan C et al (1997) Randomized trial comparing monthly low-dose leucovorin and fluorouracil bolus with bimonthly high-dose leucovorin and fluorouracil bolus plus continuous infusion for advanced colorectal cancer: a French intergroup study. J Clin Oncol 15:808-815

19. Goldberg R, Sargent DJ, Morton RF et al (2004) A randomized controlled trial of fluorouracil plus leucovorin, irinotecan, and oxaliplatin combinations in patients with previously untreated metastatic colorectal cancer. J Clin Oncol 22:23-30

20. Cheeseman S, Joel S, Chester J et al (2002) A "modified de Gramont" regimen of fluorouracil, alone and with oxaliplatin, for advanced colorectal cancer. Br J Cancer 87:393-399
21. Andre T, Louvet C (1999) Maindrault-Goebel F et al CPT-11 (irinotecan) addition to bimonthly, high-dose leucovorin and bolus and continous-infusion 5-fluorouracil (FOLFIRI) for pretreated metastatic colorectal cancer. Eur J Cancer 35:1343-1347

22. Merkel S, Bialecki D, Meyer T et al (2009) Comparison of clinical risk scores predicting prognosis after resection of colorectal liver metastases. J Surg Oncol 100:349-357

23. Bockhorn M, Sotiropoulos GC, Sgourakis G et al (2009) Major liver resections in the elderly-is an aggressive approach justified? Int J Colorectal Dis 24:83-86

24. Xu J, Wei Y, Zhong Y et al (2009) Hepatectomy for liver metastasis of colorectal cancer. Int J Colorectal Dis 24:419-425

25. Gill S, Loprinzi CL, Sargent DJ et al (2004) Pooled analysis of fluorouracil-based adjuvant therapy for stage II and III colon cancer: who benefits and by how much? J Clin Oncol 22:1797-1806

26. Kemeny N, Huang Y, Cohen AM et al (1999) Hepatic arterial infusion of chemotherapy after resection of hepatic metastases from colorectal cancer. N Engl J Med 341:2039-2048

27. Alberts SR, Roh MS, Mahoney MR et al (2010) Alternating systemic and hepatic artery infusion therapy for resected liver metastases from colorectal cancer: a North Central Cancer Treatment Group (NCCTG)/National Surgical Adjuvant Breast and Bowel Project (NSABP) Phase II Intergroup Trial, N9945/CI66. J Clin Oncol 28:853-858 